\section{Musculoskeletal examination skills of pediatric residents}

\author{
Roman Jurencak, Johannes Roth \\ Division of Rheumatology, Children's \\ Hospital of Eastern Ontario \& University \\ of Ottawa, Ottawa, Canada
}

\section{Abstract}

The aim of our work is to assess musculoskeletal examination skills of pediatric residents. A self-assessment questionnaire with five-point Likert scale (1=strongly disagree, $5=$ strongly agree) was used. After completion of questionnaires, the residents were taught proper joint examination techniques. Thereafter, the residents were asked to judge whether they had rated their skills accurately. The session was attended by 25/41 residents (61\%). Overall, their reported examination skills were poor with a mean score of 3.0 for PGY1, 2.1 for PGY2, 2.2 for PGY3 and 3.0 for PGY4. After being taught the proper joint examinations techniques, $22 \%$ of residents reported they initially overestimated their skills and 5\% underestimated their skills. The residents felt most comfortable with the knee exam, least comfortable with the finger and wrist joint exam. Most pediatric residents did not feel they can perform a focused musculoskeletal exam.

\section{Introduction}

Musculoskeletal (MSK) symptoms in children are common, occurring in up to $30 \%$ of children. ${ }^{1-3}$ The differential diagnosis is quite broad and competent MSK examination skills are crucial for accurate patient assessment, particularly because MSK symptoms are not always easily volunteered by children, and parental observations may be vague. In addition, diseases like juvenile idiopathic arthritis (JIA) most commonly present with abnormal gait and joint swelling rather than reported pain. ${ }^{4}$ It is important to identify children with rheumatic disorders such as JIA as the disease is a common cause of chronic disability and joint damage can occur early. ${ }^{5,6}$ Prompt treatment is necessary to improve functional outcome, however, this relies on early diagnosis and prompt referral to a specialist's care. ${ }^{7,8}$ Unfortunately, delay in receiving pediatric rheumatology services often hinders state-ofthe-art treatment of children with JIA and leads to adverse outcomes. ${ }^{9}$ It was our perception that pediatric residents do not always demonstrate sufficient skills in this regard.
The aim of this study was to assess whether pediatric residents can perform an MSK exam focused on the assessment of inflammatory changes in joints.

\section{Materials and Methods}

\section{Study group}

All pediatric residents attending Academic Half Day at our institution.

\section{Assessment}

A self-assessment questionnaire was handed out to all residents to assess their MSK examination skills. First, the residents were asked whether they can perform a full MSK exam focused on presence/absence of arthritis. Thereafter, they were asked whether they can examine specific joints (fingers/toes, wrists, elbows, shoulders, hips, knees, ankles, and spine). Responses were scored using a fivepoint Likert scale ( $1=$ strongly disagree, $5=$ strongly agree). After completion of questionnaires, the residents were taught proper joint examination techniques by an experienced rheumatologist (RJ). Upon completion of the module, the residents were asked to judge whether they rated their skills accurately in the previous self-assessment questionnaire, using a three-point scale (Underestimated, Accurate and Overestimated).

\section{Results}

The session was attended by 25 out of 41 residents (61\%) at our institution. Sixteen residents were absent because of on-service duties, post call leave or holidays. The questionnaire was filled out by all attendees of the Academic Half Day and the response rate and completeness were $100 \%$. Overall, the residents reported insufficient MSK exam skills with a mean score of 3.0 for PGY1, 2.1 for PGY2, 2.2 for PGY3 and 3.0 for PGY4. Moreover, $22 \%$ of residents initially overestimated their skills while only $5 \%$ underestimated their skills (Table 1). The residents felt most comfortable with the knee exam (mean score 3.2), least comfortable with the finger and wrist joints exam (mean score 2.4 and 2.5, resp., Table 2).

Out of these 25 residents, 23 did complete a rotation in orthopedics prior to this study. Only 2 residents completed an elective rotation in rheumatology and both of them were in their fourth year of training (PGY4). The average score of these two students was 4.0.
Correspondence: Roman Jurencak, Division of Rheumatology, Children's Hospital of Eastern Ontario \& University of Ottawa, 401 Smyth Road, Ottawa, K1H 8L1, Canada.

Tel. +1.613.737 7600 - Fax: +1.613.738.4297.

E-mail: rjurencak@cheo.on.ca

Key words: postgraduate education, physical examination,musculoskeletal system, pediatrics

Contributions: RJ, study design, data acquisition, data analysis and interpretation, article creation; JR, study design, data analysis and interpretation, article revision.

Conflict of interest: the authors report no conflicts of interest.

Received for publication:18 August 2011.

Revision received: 7 October 2011.

Accepted for publication: 3 November 2011.

This work is licensed under a Creative Commons Attribution 3.0 License (by-nc 3.0).

CC Copyright R. Jurencak and J. Roth., 2011

Licensee PAGEPress, Italy

Rheumatology Reports 2011; 3:e11

doi:10.4081/rr.2011.e11

\section{Discussion}

This study shows overall suboptimal musculoskeletal examination skills of pediatric residents regardless of their postgraduate year of training. This is consistent with other reports. ${ }^{10,11}$ which implies that there needs to be more attention paid to teaching a proper MSK exam in the postgraduate training.

In our study, residents in their first year of training achieved highest scores. Thereafter there was an apparent decline in their reported MSK exam skills. At our institution, the rotation in pediatric rheumatology is optional and has been in place only since January 2010; at the time of this study only two of our core residents had completed the rotation. These two PGY4 residents achieved the highest scores in the questionnaire and their results skewed somewhat the score of the PGY4 group. If these two residents were excluded, the average score of the PGY4 group was only 2.0. Even though it is difficult to draw any firm conclusions from these small numbers, it appears that their exposure to rheumatology may have resulted in a better set of skills for performing a competent MSK exam.

Interestingly, 23 out of 25 residents completed a rotation in orthopedics, but did not report having good skills to perform a focused MSK exam. This may be due to the fact that orthopedics is a surgical subspecialty not necessarily focusing on ruling out arthritis. Rather, 
patients are often referred to a rheumatologist in the absence of a clear surgical/orthopedic diagnosis for further assessment. Therefore, it seems that MSK examination skills to assess inflammatory changes of joints are not acquired during orthopedic rotation.

This study reports the results of residents' self assessment. While this method of knowledge and skill evaluation may not always be accurate, with some evidence suggesting that clinicians tend to under-report their skill level, ${ }^{12}$ the results of this study reflect our perception of residents' MSK exam skills. Moreover, the design of this survey was unique by allowing responders to assess whether their self-evaluation had been accurate after receiving formal instructions on proper techniques of MSK exam. The data show that almost a quarter of the residents felt they initially overestimated their joint examination skills.

The Academic Half Day is a mandatory teaching session and therefore our study population is unlikely to be biased toward those with an awareness of their learning needs in MSK exam.

The use of a non-validated assessment tool is a potential limitation of this study. We did not establish the validity and reliability of the simple self assessment questionnaire; rather the students were asked to judge the accuracy of theirs answers once they were taught the proper examination techniques. The assessment tool was used to document subjective perception of resident's own musculoskeletal examination skills. It was not used as a measurement of efficacy of an intervention. We feel that under these circumstances the use of a non-validated questionnaire is acceptable; however, future studies are needed to confirm our conclusions.

Our conclusion from these data is that pediatric residents have suboptimal MSK examination skills and the current situation needs to be improved. However, with rising requirements for aspiring young doctors and overfilled curriculums, there are significant, often conflicting, pressures on educators. The first step toward improvement may be to define learning outcomes of the MSK exam teaching. It can then be decided how to effectively amend the existing curriculums in order to deliver and assess appropriate MSK exam skills. At least brief hands-on workshops and bed-side teaching should be dedicated to teaching of a full MSK exam. Routine use of educational tools such as the pediatric adaptation of the adult GALS (Gait, Arms, Legs and Spine) should be encouraged as it was found to be sensitive, quick to perform and acceptable to children and parents. ${ }^{13}$ Future studies are needed to

Table 1. Self reported evaluation of musculoskeletal (MSK) exam skills

\begin{tabular}{lcccc} 
& $\begin{array}{c}\text { Overall good } \\
\text { MSK exam skills* }\end{array}$ & Underestimated & Accurate & Overestimated \\
PGY 1 $(n=7)$ & 3.0 & $0 \%$ & $85 \%$ & $15 \%$ \\
PGY 2 (n=8) & 2.1 & $0 \%$ & $88 \%$ & $12 \%$ \\
\hline PGY 3 (n=5) & 2.2 & $0 \%$ & $40 \%$ & $60 \%$ \\
PGY 4 (n=5) & 3.0 & $20 \%$ & $80 \%$ & $0 \%$ \\
\hline Average score & 2.6 & $5 \%$ & $73 \%$ & $22 \%$ \\
\hline
\end{tabular}

${ }^{*}$ Mean score of rating scale where $1=$ strongly disagree, $5=$ strongly agree.

Table 2. Joint specific scores.

\begin{tabular}{lccccc}
$\begin{array}{l}\text { Can you examine } \\
\text { the following joints: }\end{array}$ & $\begin{array}{c}\text { Mean scores of reported joint examinations skills * } \\
(\mathrm{n}=7)\end{array}$ & $\begin{array}{c}\text { PGY 2 } \\
(\mathrm{n}=8)\end{array}$ & $\begin{array}{c}\text { PGY 3 } \\
(\mathrm{n}=5)\end{array}$ & $\begin{array}{c}\text { PGY 4 } \\
(\mathrm{n}=5)\end{array}$ & Average \\
Fingers/toes & 3.1 & 2.0 & 2.0 & 2.3 & 2.4 \\
Wrist & 3.1 & 2.3 & 2.4 & 2.3 & 2.5 \\
\hline Elbow & 3.0 & 2.5 & 3.0 & 2.3 & 2.7 \\
Shoulder & 3.1 & 2.9 & 2.8 & 3.0 & 3.0 \\
\hline Hip & 3.3 & 2.6 & 3.0 & 2.3 & 2.8 \\
Knee & 3.4 & 3.0 & 3.4 & 3.0 & 3.2 \\
\hline Ankle & 3.0 & 2.6 & 3.0 & 3.0 & 2.9 \\
Spine & 3.0 & 2.4 & 3.6 & 2.7 & 2.9 \\
\hline
\end{tabular}

*Mean score of rating scale where $1=$ strongly disagree, $5=$ =strongly agree.

evaluate whether these measures will have a positive effect on clinical MSK exam skills, improve performance in clinical practice and ultimately lead to early diagnosis, care and better outcomes for children with inflammatory MSK disorders.

\section{References}

1. De Inocencio J. Epidemiology of musculoskeletal pain in primary care. Arch Dis Child 2004;89:431-4.

2. Goodman, JE, McGrath PJ. The epidemiology of pain in children and adolescents: a review. Pain 1991;46:247-64.

3. Symmons DP, Jones M, Osborne J et al. Pediatric rheumatology in the United Kingdom: data from the British Pediatric Rheumatology Group National Diagnostic Register. J Rheumatol 1996;23:1975-80.

4. McGhee JL, Burks FN, Sheckels JL, Jarvis JN. Identifying children with chronic arthritis based on chief complaints: absence of predictive value for musculoskeletal pain as an indicator of rheumatic disease in children. Pediatrics 2002;110:354-9.

5. Levinson JE, Wallace CA. Dismantling the pyramid. J Rheumatol Suppl 1992;33:6-10.

6. Wallace CA, Levinson JE. Juvenile rheumatoid arthritis: outcome and treat- ment for the 1990s. Rheum Dis Clin North Am 1991;17:891-905.

7. Sherry DD, Stein LD, Reed AM et al. Prevention of leg length discrepancy in young children with pauciarticular juvenile rheumatoid arthritis by treatment with intraarticular steroids. Arthritis Rheum 1999;42:2330-4.

8. Flato B, Aasland A, Vinje 0, Forre 0 . Outcome and predictive factors in juvenile rheumatoid arthritis and juvenile spondyloarthropathy. J Rheumatol 1998;25:36675.

9. Manners PJ. Delay in diagnosing juvenile arthritis. Med J Aust 1999;171:367-9.

10. Hergenroeder AC, Chorley JN, Laufman L, Fetterhoff AC. Pediatric residents' performance of ankle and knee examinations after an educational intervention. Pediatrics 2001;107:E52.

11. Jandial S, Myers A, Wise E, Foster HE. Doctors likely to encounter children with musculoskeletal complaints have low confidence in their clinical skills. J Pediatr 2009;154:267-71.

12. Cohen L, Manion L, Morrison K. Research Methods in Education. Routledge, New York; 2007.

13. Foster HE, Kay LJ, Friswell M et al. Musculoskeletal screening examination (pGALS) for school-age children based on the adult GALS screen. Arthritis Rheum 2006;55:709-16. 